\title{
Revisión bibliográfica de cáncer vesical de origen laboral
}

\section{Literature review of occupational bladder cancer}

\author{
Beatriz Suárez-Baena ${ }^{1,4}$, Nikaury Encarnación-Encarnación ${ }^{2,4}$, Beatriz Valladares-Lobera ${ }^{3,4}$ \\ 1. Hospital Universitario Central de Asturias (HUCA). Oviedo. UDMT de Asturias. España. \\ 2. IBERMUTUAMUR Oviedo. UDMT de Asturias. España. \\ 3. Hospital de Cabueñes. Gijón. UDMT de Asturias. España. \\ 4. Unidad Docente de Medicina del Trabajo de la Comunidad de Madrid. Madrid. España.
}

Recibido: 27-03-15

Aceptado: 06-04-15

\section{Correspondencia}

Beatriz Suárez-Baena

C/ Quirinal, n. ${ }^{\circ} 15,2^{\circ}$ B

C.P. 33401 . Avilés. Asturias. España.

Teléfono: 647707429

Correo electrónico: b_sba@hotmail.com

Este trabajo se ha desarrollado dentro del Programa Científico de la Escuela Nacional de Medicina del Trabajo del Instituto de Salud Carlos III en convenio con Unidad Docente de Medicina del Trabajo de la Comunidad de Madrid.

Resumen

Introducción y Objetivos: Realizamos una revisión bibliográfica para determinar la relación entre la exposición a determinadas sustancias en el ámbito laboral y el desarrollo de cáncer vesical, clarificar aquellas profesiones con mayor riesgo y si éste presenta diferencias entre géneros.

Material y Métodos: Revisión sistemática. Bases de datos: MEDLINE (Medlars Online International Literature, vía PubMed), OSH UPDATE, IBECS e ILO (International Labour Organization).Se incluyeron los artículos publicados en Inglés o Español, estudios Internacionales o Nacionales, publicaciones dirigidas a relacionar efectos de exposición laboral con cáncer vesical, en los últimos 5 años (2009-2014).

Resultados: Se localizaron 156 estudios. Sólo 18 cumplieron los criterios de inclusión. Se encontraron 3 estudios de cohortes, 9 de casos y controles, 3 meta-análisis, 2 estudios descriptivos y una serie de casos. Las principales profesiones de riesgo son las relacionadas con la industria de hierro, acero y colorantes, minería de carbón, trabajadores del caucho, pintores, agricultores, peluqueros, conductores de camiones y profesionales en contacto con sustancias explosivas.

Conclusiones: En nuestra búsqueda hemos encontrado varios estudios que evidencian relación entre la exposición laboral y el cáncer vesical, aunque se requiere más información, y de más calidad, que permita establecer resultados concluyentes.

Med Segur Trab (Internet) 2015; 61 (239) 295-310

Palabras clave: Cáncer, vejiga, laboral, ocupacional. 
Abstract

Background and objectives: We conducted a literature review to determine the relationship between exposures to certain substances in the workplace and the development of bladder cancer, clarify those professions most at risk and if this presents differences between genders.

Methods: Systematic review. Databases: MEDLINE (Medlars Online International Literature), OSH UPDATE, IBECS and ILO (International Labour Organization). Articles published in English or Spanish, Domestic and International Studies, publications aimed to relate effects of occupational exposure to bladder cancer in the last five years (2009-2014) were included.

Results: 156 studies were identified. Only 18 met the inclusion criteria. 3 cohort 9 cases and controls, 3 Meta-analyzes, 2 descriptive studies and case series were found. The main risk professions are related to the industry of iron, steel and dyes, coal mining, rubber workers, painters, farmers, hairdressers, truck drivers and professionals in contact with explosive substances.

Conclusions: In our search we found several studies showing relationship between occupational exposure and bladder cancer, even if more information is required, and better quality, to establish conclusive results.

Med Segur Trab (Internet) 2015; 61 (239) 295-310

Keywords: Bladder cancer, occupational exposure. 


\section{INTRODUCCIÓN}

Ya en el siglo XVII Bernardino Ramazzini efectuó los primeros estudios sobre salud ocupacional, haciendo referencia a "micciones sanguinolentas» en los capítulos de las Enfermedades de los químicos y en Enfermedades de "obreros que trabajan a pie», al hablar de los pintores de construcciones en su obra "De morbis artificum diatriba" ${ }^{1}$.

Una de las primeras referencias en cuanto a la relación entre cáncer y actividad laboral la encontramos en 1775, cuando Percival Pott describe en Inglaterra el cáncer de escroto en jóvenes limpiadores de chimeneas, sugiriendo una relación causal entre este tipo de cáncer y el hollín.

En 1895, Ludwing Rehm comunicó casos de cáncer vesical en trabajadores de una fábrica alemana de anilinas. Esta observación llevó a estudios similares en muchos otros países y al reconocimiento de la causalidad de las aminas aromáticas en el cáncer de vejiga humano. La Organización Internacional del Trabajo (OIT) indicó, en 1921, que la bencidina y la naftilamina eran los carcinógenos responsables y lanza la primera alerta en materia de salud laboral.

La evidencia experimental fue posterior, cuando en 1930, el patólogo Wilhelm Hueper y col. provocaron cáncer de vejiga en animales tras administrarles repetidamente Beta-naftilamina.

En 1970, se verifican 36 muertos por tumores vesicales en los obreros de la Industria Piemontese dei Colori di Anilina (IPCA), una fábrica italiana de colorantes, conocida popularmente desde ese momento como «la fábrica del cancro» (la fábrica del cáncer) ${ }^{2}$.

En 1982, Carlos A. González calcula la probabilidad de padecer cáncer vesical en los trabajadores de tinte y estampado de productos textiles en Mataró (España) descubriendo los alarmantes datos de que eran casi seis veces mayor que en el resto de la población ${ }^{3}$.

En 1990 se publica el primer estudio de cáncer vesical por aminas aromáticas en China, donde su uso comenzó en la década de $1950^{4}$.

Según la American Cancer Society, el cáncer de vejiga es el cuarto en frecuencia en los países desarrollados, tras los tumores colorrectales, de pulmón y de próstata, con aproximadamente 375.000 nuevos casos al año en todo el mundo. El 65\% de los casos se registran en países desarrollados, sobre todo en el sur de Europa y Norteamérica, ligados fundamentalmente a la alta tasa de fumadores 5 .

Un $70-75 \%$ de los casos de cáncer vesical se pueden atribuir al consumo de tabaco y hasta un $20-25 \%$ están relacionados con ciertas exposiciones ocupacionales.

Diversos investigadores (Lancet, 1978; NIOSH, 1981) han calculado que entre el 23\% y el 38\% de la mortalidad por cáncer puede ser atribuida a causas ocupacionales.

Las sustancias químicas específicas relacionadas con la carcinogénesis vesical incluyen beta-naftilamina, bencidina, hidrocarburos aromáticos policíclicos (HAP) y 4-aminobifenilo. Aunque estas sustancias están prohibidas en los países occidentales, se sospecha que muchas otras sustancias químicas actualmente en uso también puedan ser causantes de dicha patología.

Las tasas más altas de cáncer de vesical se notificaron en las industrias de tinturas textiles y de caucho para neumáticos, en pintores, trabajadores del cuero, calzado, aluminio, hierro y en la industria siderúrgica.

En España se diagnostican unos 12.200 casos anuales, lo que representa el 11\% de los tumores en población masculina y el $2,4 \%$ en la femenina. La incidencia en nuestro país es de las más altas del mundo, siendo el cuarto tumor más frecuente en hombres ${ }^{6}$.

En los países árabes, y sobre todo en Egipto, la infección endémica esquistosomiasis, causada por el parásito Schistosoma haematobium, que se deposita en la vejiga de los 
pacientes originando una irritación crónica, provoca carcinoma vesical de células escamosas (SCC), poco frecuente en nuestro medio.

Se cree que los antecedentes familiares aumentan el riesgo de padecer este cáncer. En algunos casos, estos familiares pudieron haber estado expuestos al mismo químico que provoca cáncer.

Otras alteraciones, como los polimorfismos genéticos en las enzimas glutatión-stransferasa (GSTM1) y enzima N-acetiltransferasa (NAT2) (acetiladores), determinan que los organismos de los individuos sean lentos en descomponer ciertas toxinas, pudiendo provocar mayor propensión a padecer cáncer de vejiga ${ }^{7}$.

Un pequeño número de personas heredan un síndrome genético que aumenta su riesgo de cáncer de vejiga, como:

- Una mutación del gen de retinoblastoma (RB1) puede causar cáncer de ojo en niños y también aumentar el riesgo de cáncer de vejiga.

- La enfermedad de Cowden, causada por mutaciones en el gen PTEN, asociada principalmente con cánceres de seno y tiroides, tiene también mayor riesgo de cáncer de vejiga.

- El síndrome de Lynch (cáncer colorrectal hereditario no polipósico) se asocia principalmente con cáncer de colon y de endometrio, pero los pacientes afectos también tienen un riesgo aumentado de cáncer de vejiga, así como cánceres de los uréteres ${ }^{8}$.

El Instituto Sindical de Trabajo, Ambiente y Salud (ISTAS), señala el número de cánceres de vejiga (casos incidentes y muertes) atribuidos a exposiciones laborales en 2002. Las estimaciones varían entre 216 casos (estimación países nórdicos) a 2.321 casos (límite superior de estimación Steenland). Las estimaciones de número de muertes atribuidas a exposiciones laborales, varían de 71 muertes (estimación países nórdicos) a 798 muertes (límite superior de estimación Steenland). En todas las estimaciones el número de casos es bastante más inferior en mujeres que en hombres ${ }^{9}$

En España M. Kogevinas refiere 10.705 casos de cáncer de vejiga en hombres con 3.493 casos de muerte (siendo atribuidos a exposiciones laborales 428 casos de cáncer y 140 de las muertes). En mujeres se describieron 1.510 casos de cáncer vesical y 704 muertes, no asociándose ninguno a exposición laboral.

Los datos anteriormente explicados corresponden al año 2002, siendo la última referencia encontrada.

La bibliografía actual revela que la variedad histológica más frecuente en las neoplasias vesicales de origen laboral es el Carcinoma de Células Uroteliales (Uc) (que es también el tipo más frecuente en cáncer vesical no laboral), seguido a mucha distancia por Adenocarcinoma y Carcinoma de células escamosas.

La OMS, a través de la IARC (International Agency for Research on Cancer) declara que existe una evidencia fuerte entre cáncer de vejiga urinaria y los carcinógenos laborales: 4-Aminobifenilo, Aluminio y sus componentes, Auramina, Bencidina, BetaNaftilamina, Alquitrán, Magenta, Carbón gasificado y una evidencia débil con: tintes basados en Bencidina, Benzopireno, Cuero, 4-cloro-toluidina, hornos de coque, Alquitrán y Breas de alquitrán, 4-4'-Metileno bis (2-cloroanilina).

Durante 2008, 10.652 altas hospitalarias en España fueron debidas a cánceres de pulmón y de vejiga atribuibles al trabajo (se reconocieron 16 como profesionales ese mismo año). El coste del tratamiento de estos 2 tipos de cáncer ascendió a casi 88 millones de euros, de los cuales 26,5 millones correspondieron al cáncer de vejiga ${ }^{10}$.

La legislación es amplia y variada, siendo fundamental el Real Decreto 665/1997, de 12 de mayo, sobre la protección de los trabajadores contra los riesgos relacionados con la exposición a agentes cancerígenos durante el trabajo. Este Real Decreto no ha sido la 
primera normativa específica orientada a prevenir los cánceres de origen laboral, pero sí la primera referencia legal que, con posterioridad a la Ley 31/1995 de prevención de riesgos laborales, aporta los principios generales para la lucha contra los cánceres profesionales $^{11-15}$

\section{OBJETIVOS}

El objetivo principal del presente trabajo es determinar si existe evidencia científica que relacione cáncer vesical y exposición a sustancias presentes en el ámbito laboral.

Como objetivos específicos:

- Clarificar las profesiones más expuestas.

- Establecer si existe mayor sensibilidad en función del género.

- Clasificar las sustancias que están más vinculadas al cáncer vesical.

\section{MATERIAL Y MÉTODOS}

La búsqueda se llevó a cabo en las siguientes bases de datos: MEDLINE (Medlars Online International Literature, vía PubMed), OSH UPDATE, IBECS e ILO (International LabourOrganization).

Para definir los términos de la búsqueda se empleó el MeSH, considerándose adecuados "UrinaryBladderNeoplasms» como MajorTerm y "Occupational Medicine», "OccupationalExposure», «OccupationalDiseases», «OccupationalHealth», "Accidents, Occupational», "Workplace» como MeSHTerm. Se utilizaron los filtros (límites): «Humans» $\mathrm{y}$ «Last 5 years».

La ecuación de búsqueda final para su empleo en MEDLINE/PubMed quedó como sigue:((«Urinary Bladder Neoplasms»[Majr] AND «last 5 years»[PDat] AND Humans[Mesh])) AND (( «Occupational Medicine»[Mesh] OR «Accidents, Occupational»[Mesh] OR «Occupational Exposure»[Mesh] OR «Occupational Diseases»[Mesh] OR «Occupational Health»[Mesh]) OR «Workplace»[Mesh]) AND «last 5 years»[PDat] AND Humans[Mesh]).

Esta misma estrategia se adaptó a las características de cada base de datos:

En OSH UPDATE: «Occupationalexposure» $\mathrm{y}$ «Bladdercancer» como descriptores y «Urinary» en todos los campos.

En IBECS: "CANCER" (palabras) and "VEJIGA» (palabras) and "LABORAL» or «OCUPACIONAL" (palabras).

En ILO: «Urinarybladdercancer».

Se seleccionaron para su revisión los artículos que cumplieron los siguientes criterios: estudios publicados en inglés o español, estudios Internacionales o Nacionales, publicaciones dirigidas a relacionar efectos de exposición laboral con cáncer vesical, en los últimos 5 años (2009-2014).

Se excluyeron publicaciones anteriores a 2009, artículos que no aportaran información acerca del cáncer vesical de origen laboral, artículos descritos en otros idiomas distintos al inglés o español.

La principal limitación encontrada en la revisión corresponde a numerosas publicaciones relacionadas con cáncer de vejiga de causas multifactoriales.

Cuando la información dada por el abstract fue insuficiente para decidir la inclusión o exclusión de los artículos, se realizó la lectura del texto completo. 
Para la selección de los artículos se utilizaron las directrices para la publicación de estudios STROBE (Strengthening the Reporting of Observacional studies in Epidemiology) ${ }^{16}$.

Los estudios se agruparon según las variables a estudio con el fin de sistematizar y facilitar la comprensión de los resultados, considerando los siguientes datos: primer autor y año de la referencia, diseño del estudio, periodo de realización del estudio, población estudiada (incluyendo datos socio-demográficos tales como raza y sexo, agentes de exposición, tiempo de exposición, factores de confusión, resultados principales, nivel de evidencia y grado de recomendación SIGN $^{17}$ (Scottish Intercollegiate Guidelines Network).

\section{RESULTADOS}

Tras haber aplicado nuestros criterios de búsqueda, hemos recuperado 156 referencias, que tras depurar los duplicados y aplicar los criterios de exclusión y exclusión (Figura 1) fue posible recuperar 18 artículos.

Figura 1

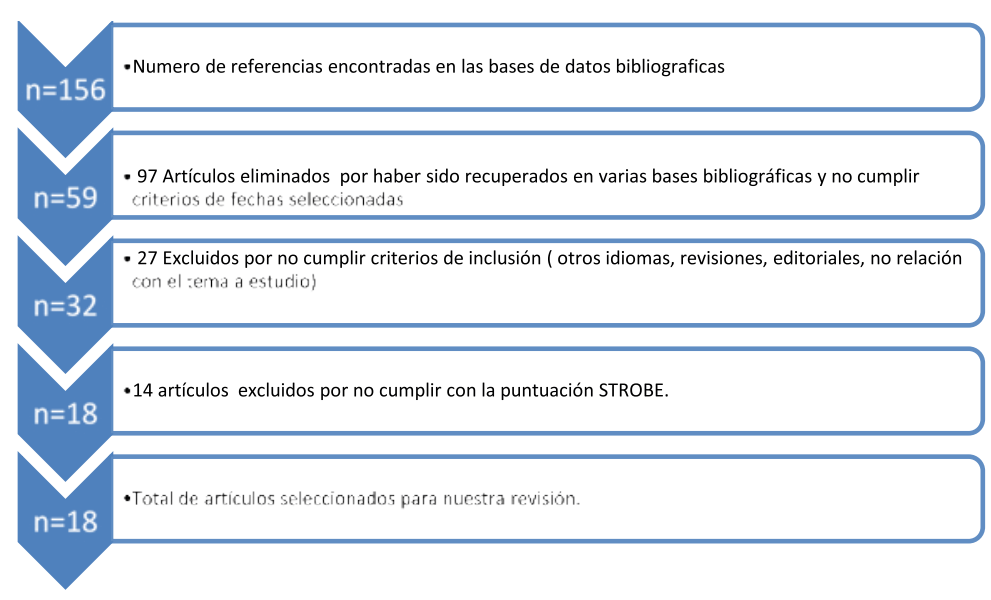

Los trabajos revisados incluyen una serie de $\operatorname{casos}^{18}, 2$ estudios descriptivos ${ }^{19,20}, 3$

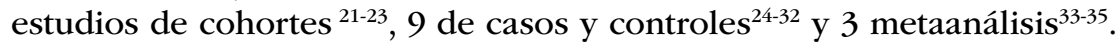

A continuación, presentamos una tabla-resumen con los datos más significativos extraídos de los artículos revisados. 

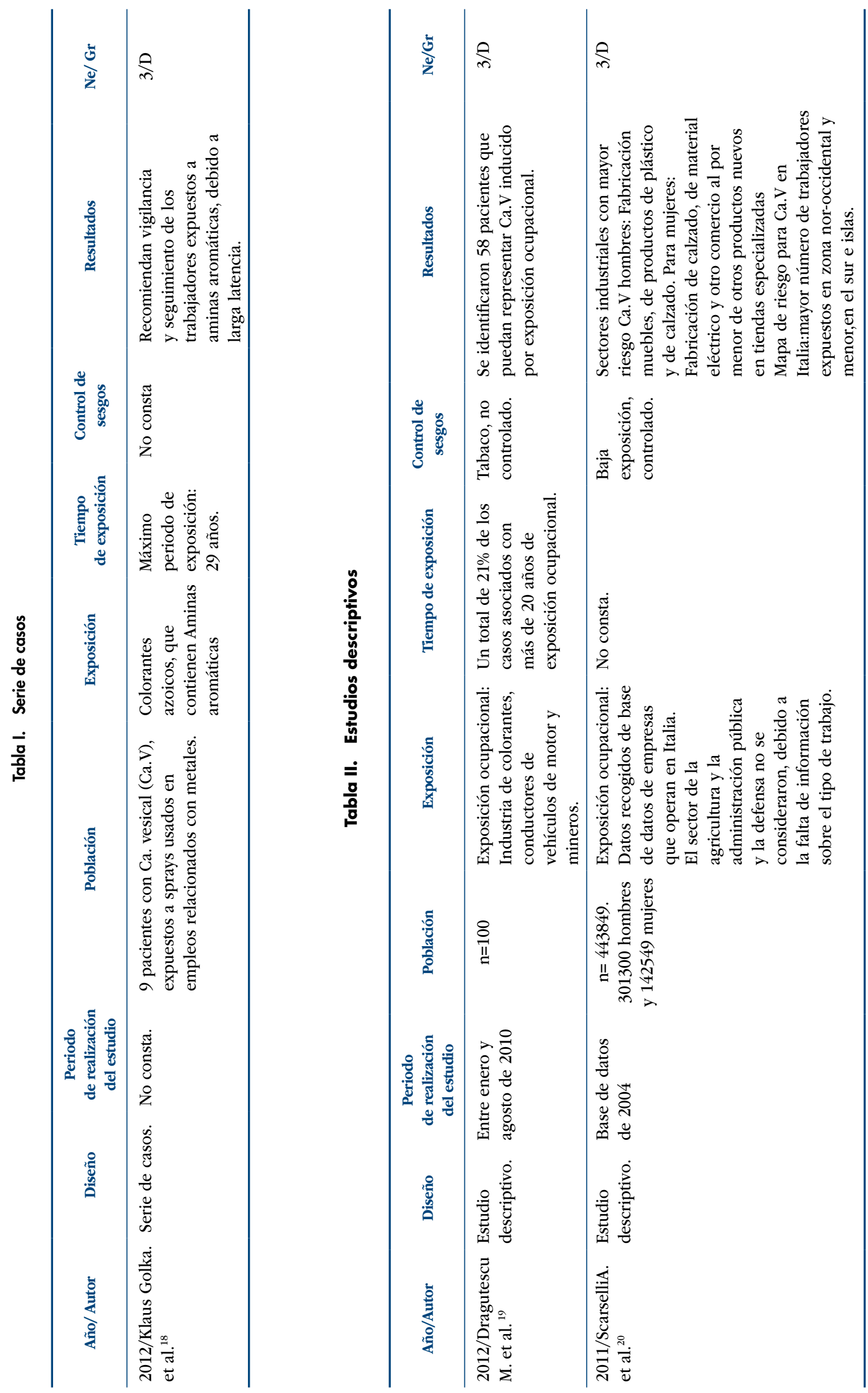


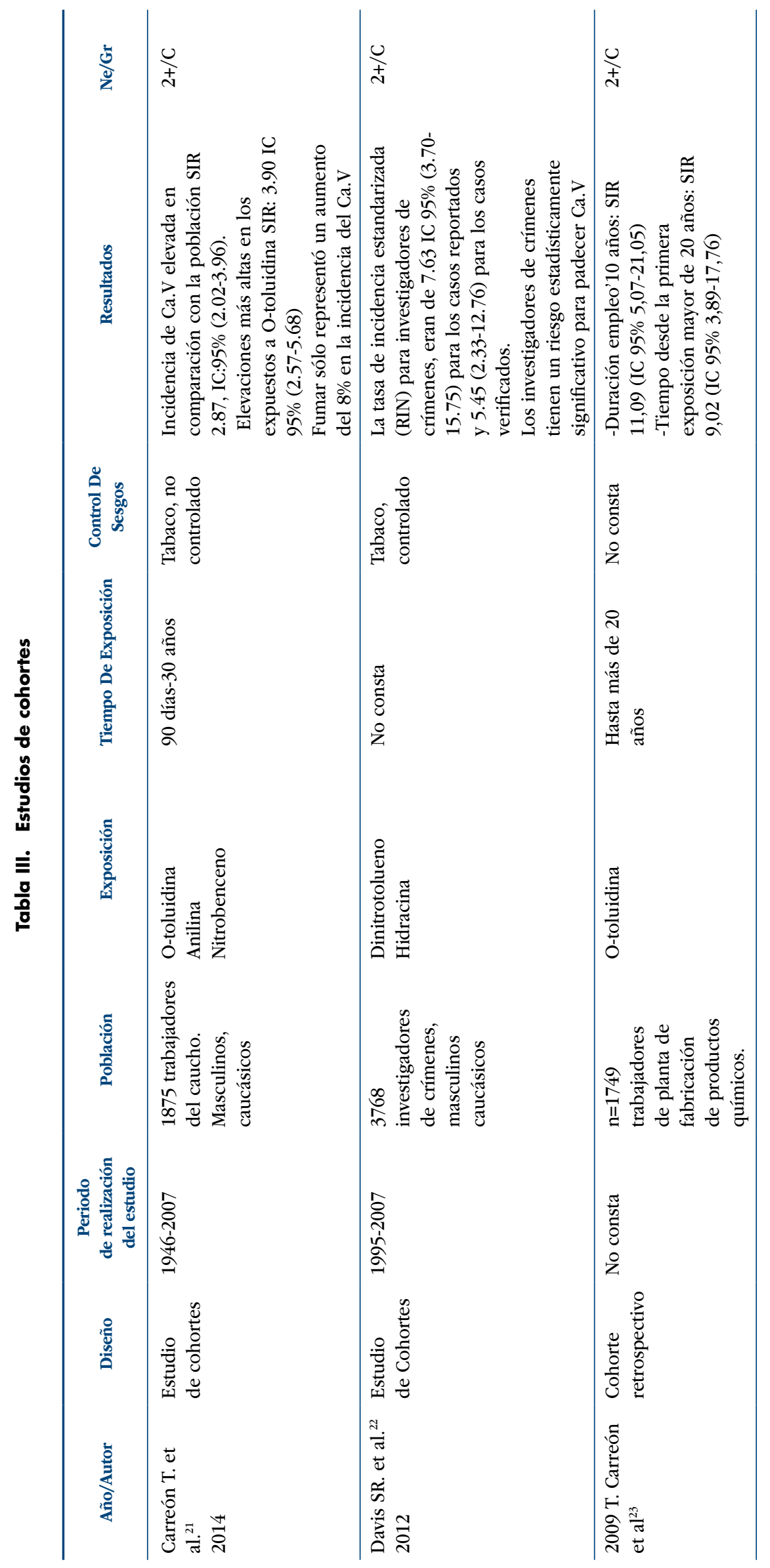




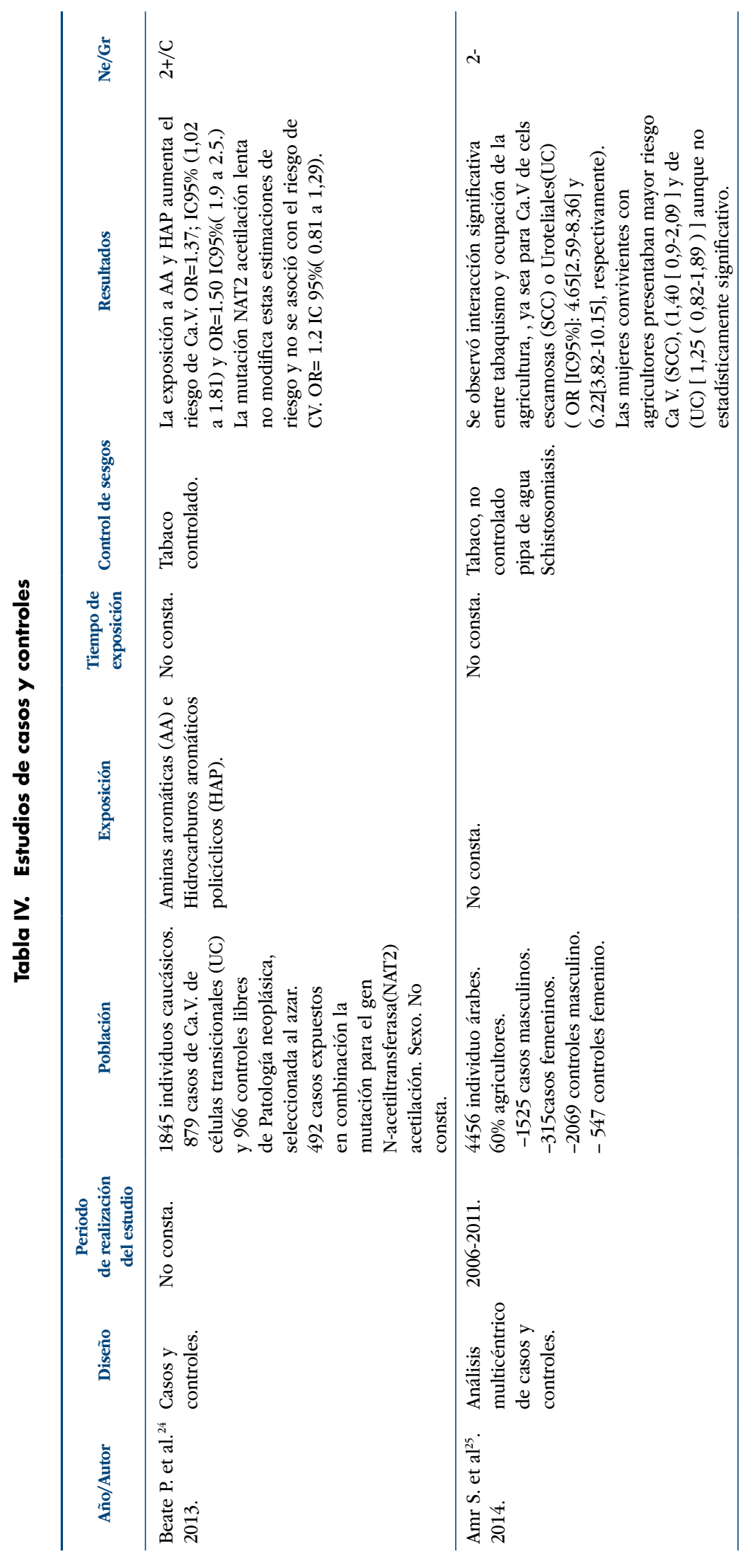




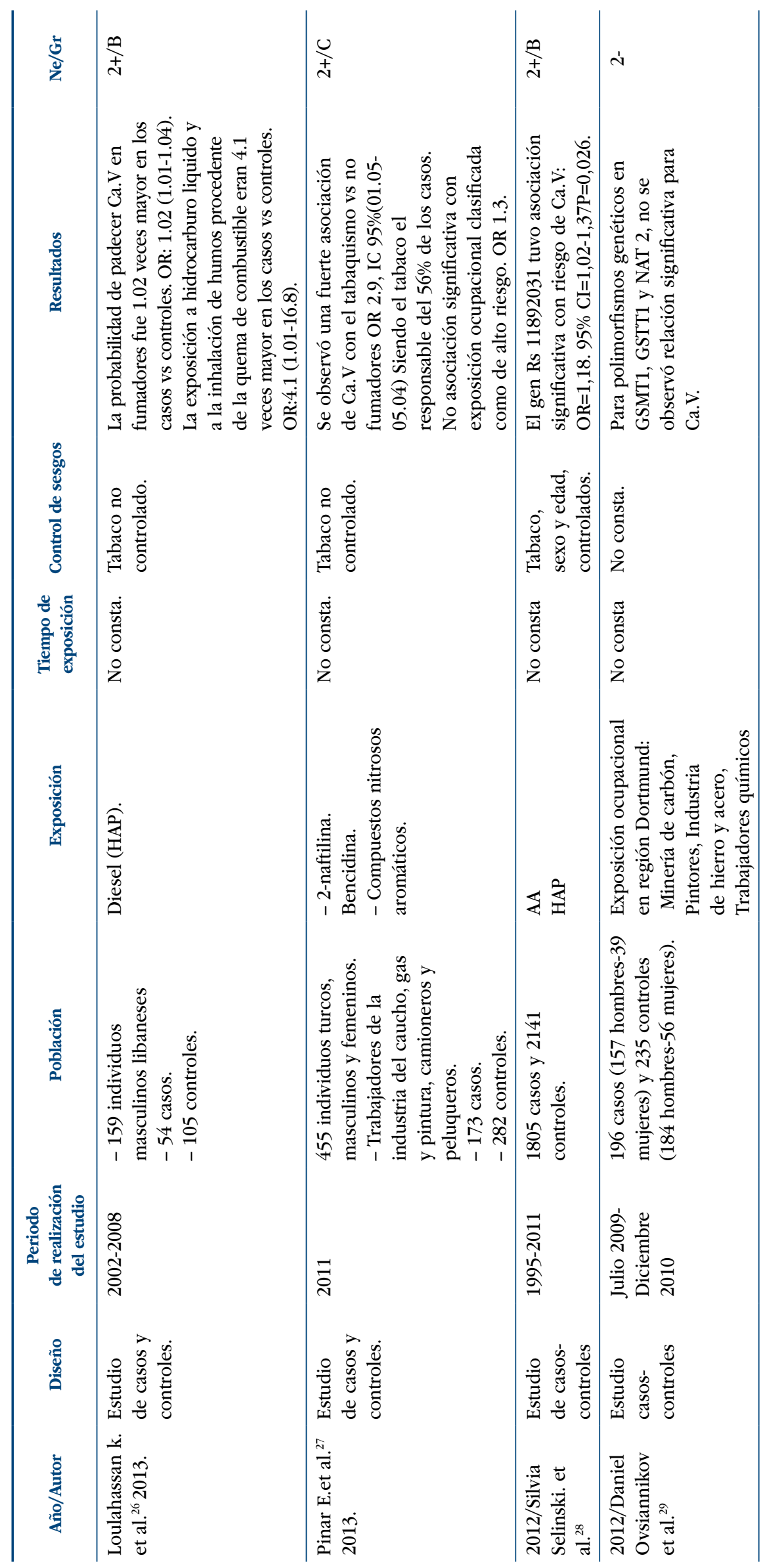




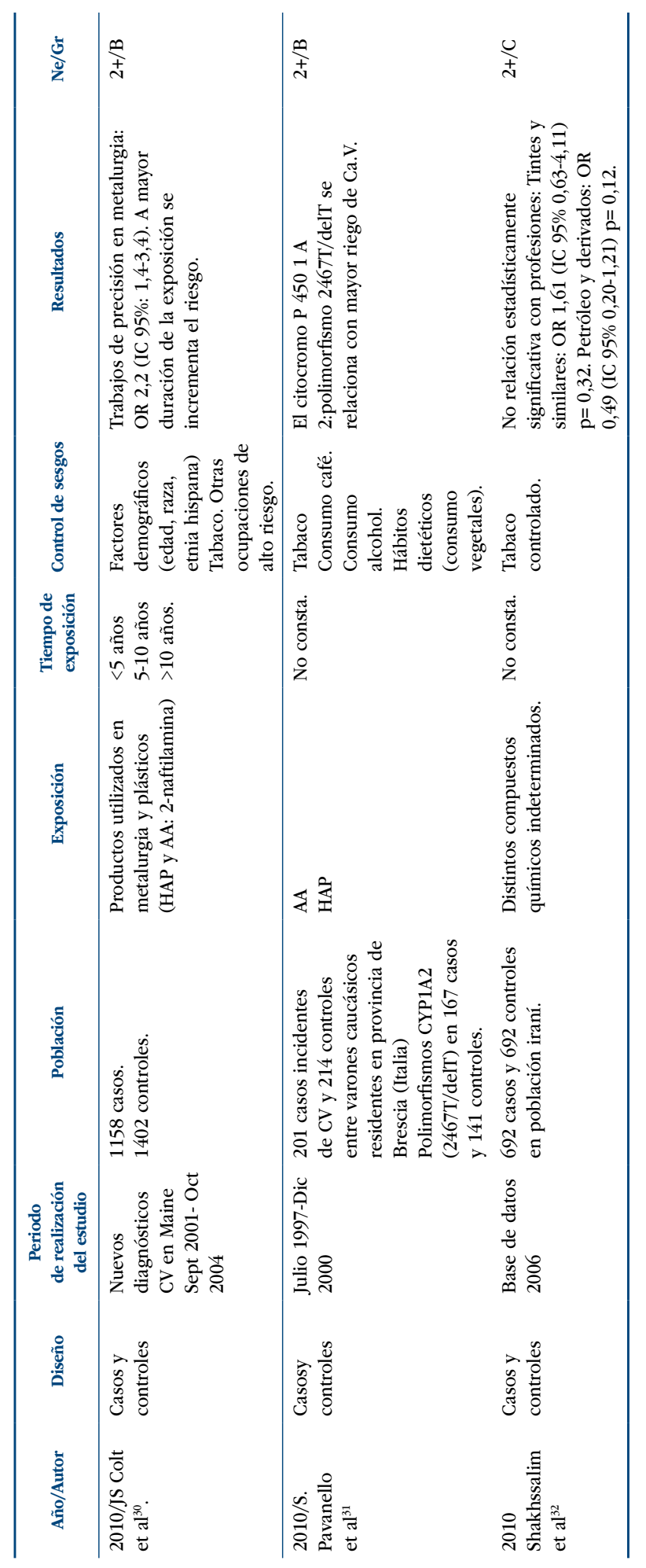




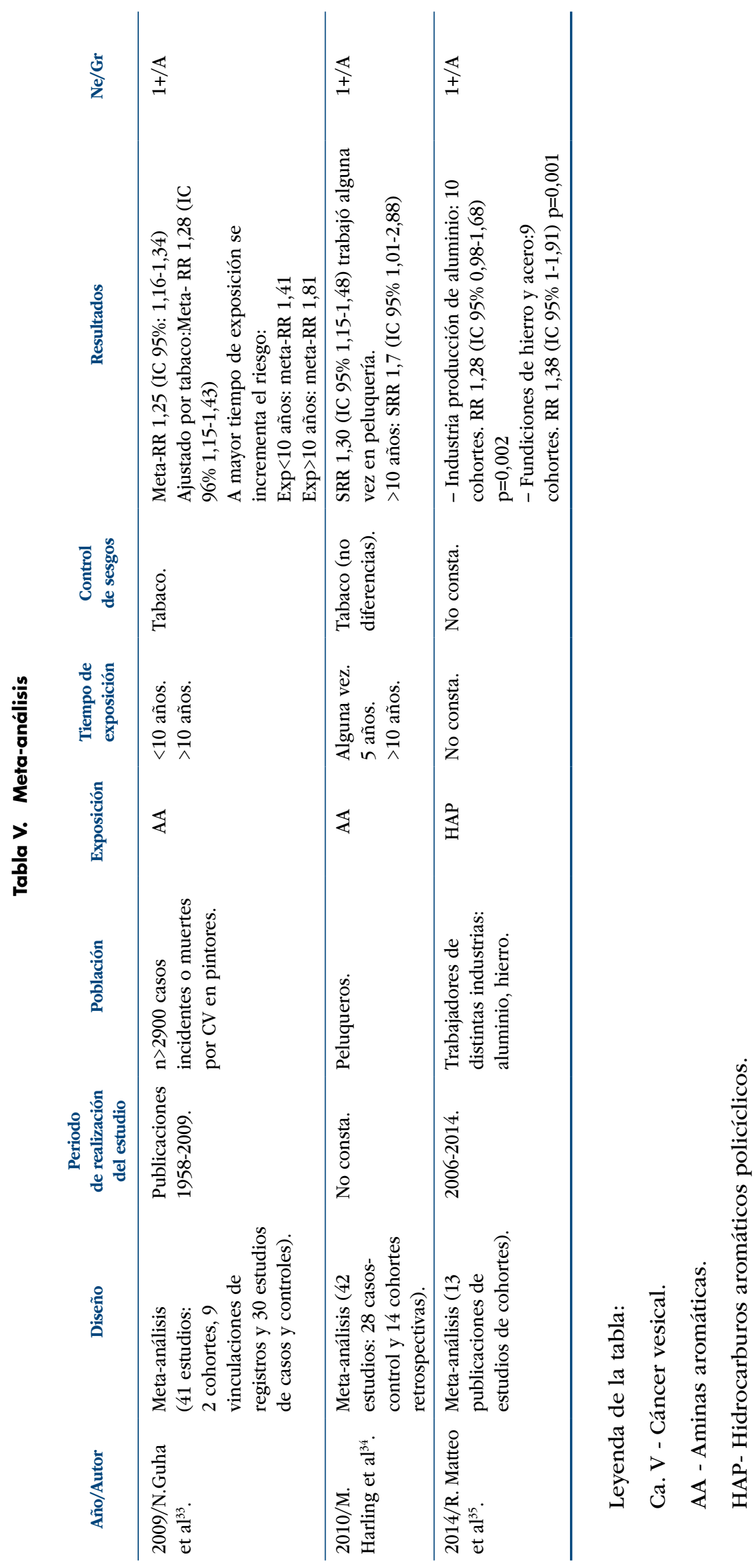


En relación a las distintas variables estudiadas en las publicaciones seleccionadas para nuestra revisión pasamos a comentar los aspectos más relevantes:

El estudio de KlausGolka ${ }^{18}$ es el que presentó menor tamaño muestral $(n=9)$, mientras que el de Scarselli A. ${ }^{20}$ fue el de mayor $(n=443849)$.

De todos los trabajos revisados, en 8 no especifican el sexo de los participantes, en 4 sólo fueron estudiados hombres y en 6 ambos géneros. Hemos observado que hay mayor prevalencia de cáncer de vejiga por exposición laboral en hombres.

Los países en los que se llevaron a cabo los estudios fueron: Alemania, Italia, Líbano, Egipto, EE.UU, Irán, Turquía.

En nuestra búsqueda hemos encontrado varios estudios que encuentran relación entre la exposición laboral y el cáncer vesical.

Sania Amr. ${ }^{25}$ examinó en Egipto la asociación entre la agricultura y el riesgo de Cáncer vesical de células escamosas, aunque sus resultados pueden ser sesgados por la infección endémica por el parásito Schistosoma Haematobium.

Dragutescu M. ${ }^{19}$ determinó la relación de Cáncer vesical en 58 trabajadores de la industria de la minería, colorantes y conductores de vehículos de motor. Scarsellii ${ }^{20}$ valoró $^{2}$ la relación entre determinadas ocupaciones con exposición a caucho y la existente relación de cáncer vesical en trabajadores de distintas empresas de Italia.

En 11 de nuestros trabajos revisados (3 estudios de cohortes, 5 estudios de casoscontroles y los 3 meta-análisis) se estudió la relación entre aminas aromáticas e hidrocarburos policíclicos y el cáncer vesical, encontrando una relación estadísticamente significativa, salvo Shakhssalim. et al. ${ }^{32}$,que no la encontraron y sugieren que los motivos podrían ser que las profesiones no se clasificaron por las sustancias químicas concretas a las que estaban expuestos.

Ovsiannikov D. ${ }^{29}$ y Beate $\mathrm{P}^{24}$ en sus estudios no consideraron significativa la relación existente entre los polimorfismos genéticos investigados, exposición ocupacional y cáncer vesical, mientras que Pavanello $S .^{31}$, sí encontró relación entre el polimorfismo genético CYP 1A2, AA, HAP y cáncer vesical.

Selinski S. ${ }^{28}$ halló relación estadísticamente significativa entre el gen Rs 11892031, cáncer vesical y la exposición ocupacional a AA Y HAP.

\section{DISCUSIÓN Y CONCLUSIONES}

Según la escala SIGN $^{17}$ (Scottish Intercollegiate Guidelines Network), los estudios seleccionados en esta revisión (serie de casos, descriptivos, cohortes, casos-controles, metaanálisis) aportan niveles de evidencia variables, desde 3 hasta 1+ (predominantemente 2+).

Por lo tanto, podemos afirmar que existe relación significativa entre cáncer vesical y determinadas profesiones expuestas a Aminas, Hidrocarburos aromáticos policíclicos. Los resultados de la presente revisión están limitados por las carencias propias del diseño de cada trabajo revisado, por ejemplo, los sesgos no controlados de tabaco ${ }^{19,21,25-27,30}$ o enfermedades endémicas ${ }^{25}$ por schistosoma.

Según nuestra revisión, las profesiones más expuestas a riesgo de cáncer vesical son las relacionadas con la industria de hierro, acero y colorantes, minería de carbón, trabajadores del caucho, pintores, agricultores, peluqueros, conductores de camiones y profesionales en contacto con sustancias explosivas.

Hemos observado predominancia del sexo masculino en las poblaciones estudiadas, probablemente atribuible a que la mayoría de los puestos en el sector industrial están ocupados por hombres. Por lo tanto, no podemos determinar evidencia científica que 
relacione el género con mayor sensibilidad a desarrollar cáncer vesical secundario a la exposición a determinadas sustancias en el ámbito laboral.

Ovsiannikov D. ${ }^{29}$ y Beate $\mathrm{P} .{ }^{24}$ en sus estudios no consideraron significativa la relación existente entre los polimorfismos genéticos investigados, exposición ocupacional y cáncer vesical, mientras que Pavanello $S .^{31}$, sí encontró relación entre el polimorfismo genético CYP 1A2, AA, HAP y Ca.V. Ante esta contradicción es probable que se abran nuevas líneas de investigación con la finalidad de realizar mapeos genéticos para identificar aquellos individuos con mayor susceptibilidad a desarrollar cáncer de vejiga por exposición a determinadas sustancias.

Entre las sustancias más relacionadas con el cáncer de vejiga ocupacional están las Aminas aromáticas (Anilina, O-Toluidina, Bencidina, 4-aminobifenilo, Magenta) y los Hidrocarburos aromáticos policíclicos (Dinitrotolueno). También hemos inferido que mayor tiempo de exposición se relaciona con mayor riesgo de cáncer vesical. Esto concuerda con trabajos previamente realizados en los que se evidenció la causalidad de estas sustancias en el cáncer vesical.

Nuestra conclusión tras haber revisado los estudios seleccionados, varios con máximo grado de evidencia (3, meta-análisis), es que existe vinculación probada entre exposición ocupacional a Aminas aromáticas e Hidrocarburos policíclicos y cáncer de vejiga.

No obstante, consideramos aconsejable realizar más estudios para conseguir más información que permita establecer resultados concluyentes sobre relación de este cáncer y otras exposiciones laborales, así como vigilancia y seguimiento de los trabajadores expuestos a las sustancias ya confirmadas.

En este sentido ya hay proyectos en marcha, como el cuestionario ${ }^{36}$ desarrollado por $^{-}$ el Centro de Investigación de Leibniz de medio ambiente de trabajo y los factores humanos (IfADo), como un proyecto del plan de acción mundial en el marco de la OMS, con la finalidad de determinar la exposición a sustancias potencialmente cancerígenas en la historia laboral de pacientes con cáncer de vejiga.

\section{REFERENCIAS BIBLIOGRÁFICAS}

1. http://bvs.sld.cu/revistas/rst/vol13_3_12/rst07312.htm Ramazzini, "De morbisartificum diatriba"

2. Benedetto P, Masselli G, Spagnoli U, Terracini B. La Fabbrica Del Cancro L'IPCA Di Ciriè. Torino: Einaudi; 1976

3. González CA. Ocupación, trabajo, café, y cáncer de vejiga en la Comarca del Maresme. Barcelona: Universidad Autónoma de Barcelona; 1983.

4. Rodríguez CA. No aceptar cancerígenos. Por Experiencia [Internet] 2001 [citado 1 feb 2013]; Disponible en: http://www.porexperiencia.com/articulo.asp?num=14\&pag=12\&titulo=Noaceptarcancerigenos

5. http://www.cancer.org/español/cancer/cancerdevejiga/guiadetallada/cancer-de-vejiga-causes-risk-factor.

6. https://www.aecc.es/sobreelcance/cancerporlocalizacion/cancerdevejiga/paginas/incidencia.

7. j.ferris,J.garcia,o. Berbel y J.A. ortega. Factores de riesgo constitucionales y ocupacionales asociados al cáncer vesical. Revisión sistemática. Actas Urol Esp.2013;37 513-522.

8. Ferrís J, Garcia J, Berbel O, Ortega JA. Constitutional and occupational risk factors associated with bladder cancer.ActasUrol Esp. 2013 Sep;37(8):513-22. doi: 10.1016/j.acuro.2013.01.001. Epub 2013 May 9.

9. http://www.istas.net/web/abreenlace.asp?idenlace $=2207$

10. http://scielo.isciii.es/scielo.php?script=sci_arttext\&pid=S1135-57272012000200002\&lng=pt\&nrm=iso

11. Real Decreto 1299/2006, de 10 de noviembre, por el que se aprueba el cuadro de enfermedades profesionales en el sistema de la Seguridad Social y se establecen criterios para su notificación y registro.

12. Real Decreto 665/1997, de 12 de mayo, sobre la protección de los trabajadores contra los riesgos relacionados con la exposición a agentes cancerígenos durante el trabajo. Modificado por R.D. 1124/2000 y R.D. 349/2003.

13. Real Decreto 374/2001, sobre la protección de la salud y seguridad de los trabajadores contra los riesgos relacionados con los agentes químicos durante el trabajo. 
14. Real Decreto 363/1995, por el que se aprueba el Reglamento sobre notificación de sustancias nuevas y clasificación, envasado y etiquetado de sustancias peligrosas (incorporación de la Directiva del Consejo 67/548/CEE).

15. Real Decreto $255 / 2003$, por el que se aprueba el Reglamento sobre clasificación, envasado y etiquetado de preparados peligrosos.

16. [http://strobe-statement.org/].Von ELM E.AltmanDG,EggerM,Pockock SJ, Gotzsche PC Van-denbroukeJ. Et al. Declaracion de la iniciativa STROBE (STrengtheningtheReporting of Observacional studies in Epidemiology) directrices para la comunicación de estudios observacionales. Gac Sanit. 2008;22(2):144150

17. http://www.sign.ac.uk/pdf/SIGN141.pdf

18. Golka $\mathrm{K}^{1}$, Kopps S, Prager HM, Mende Sv, Thiel R, Jungmann O, Zumbe J, Bolt HM, Blaszkewicz M, Hengstler JG, SelinskiS.Bladder cancer in crack testers applying azo dye-based sprays to metal bodies.J ToxicolEnvironHealth A. 2012;75(8-10):566-71. doi: 10.1080/15287394.2012.675309

19. Drăgutescu M. ${ }^{1}$, Geavlete B, Mul冈escu R, Mihai B, Moldoveanu C, GeavleteP.The influence of toxic working environment on the urothelial bladder tumors characteristics, the experience of "Sf. Ioan" Clinical Emergency Hospital on selected series.J Med Life. 2012 Sep 15;5(3):304-7. Epub 2012 Sep 25.

20. Scarselli $\mathrm{A}^{1}$, Scanno P, Marinaccio A, Iavicoli S Bladder cancer and occupational exposure: estimating the workers potentially at risk in Italy.Ann IstSuper Sanita. 2011;47(2):200-6. doi: 10.4415/ANN_11_02_12.

21. Tania C., Misty J H. Kevin W.H. Bladercáncer incidence among workers exposed to O- toluidine, aniline, and nitrobenzene at a rubber chemical manufacturing plant. Cohort study.Occup Environ Med.2014; 71: 175-182.

22. Susan R. Davis. Xuguang T. Edward J. Bernacki. Evaluation of a bladder cáncer cluster in a population of criminal ivestigatorswiht the bureau of alcohol, tobacco firearms and explosives.Journal of Enviromental and public health.Vol 2013. Article ID986023,10 pages.

23. Increased bladder cancer risk among workers exposed to o-toluidine and aniline: a reanalysis.Carreón T, Hein MJ, Viet SM, Hanley KW, Ruder AM, Ward EM.Occup Environ Med. 2010 May;67(5):348-50. doi: 10.1136/oem.2009.051136. Epub 2009 Nov 2.

24. Pesch B ${ }^{1}$, Gawrych K, Rabstein S, Weiss T, Casjens S, Rihs HP, Ding H, Angerer J, Illig T, Klopp N, Buenode-Mesquita B, Ros MM, Kaaks R, Chang-Claude J,Roswall N, Tjønneland A, Overvad K, Clavel-Chapelon F, Boutron-Ruault MC, Dossus L, Boeing H, Weikert S, Trichopoulos D, Palli D, Sieri S, TuminoR,Panico S, Quirós JR, González C, Sánchez MJ, Dorronsoro M, Navarro C, Barricarte A, Ljungberg B, Johansson M, Ulmert D, Ehrnström R, Khaw KT, Wareham N, Key TJ, Ferrari P, Romieu I, Riboli E, Brüning T, Vineis P. N-acetyltransferase 2 phenotype, occupation, and bladder cancer risk; Cancer Epidemiol Biomarkers Prev. 2013 Nov;22(11):2055-65. doi: 10.1158/1055-9965.EPI-13-0119-T. Epub 2013 Oct 3.

25. Sania Arm Rebecca D.,Doa A., Saleh M., LAurence S., MagderNabiel N., Mikhail, Diane M.,Agricultural Workers and urinary bladder cancer risk in Egypt. Archives of Enviroment and occupational health.Vol 69, No. 1, 2014.

26. LoulouHassan K., Ibrahim Adnan Y., Michel elias J. Mohamad Ahmad M., Hassan Rida D. Urinary bladder cancer Risk factors: A lebanese case-control study. Asian Pacific J cancer Prev; 14(5) 3205-3211.

27. Pinar E., Beyhan C, Eva Negric. Smoking, occupation, history of selected disease and bladder cancer risk in manisa.Eur journal of cancer Prev. (2014. vol 23,, 1 pag 58-61.

28. Selinski $S^{1}$, Lehmann ML, Blaszkewicz M, Ovsiannikov D, Moormann O, Guballa C, Kress A, Truss MC, Gerullis H, Otto T, Barski D, Niegisch G, Albers P, Frees S, Brenner W, Thüroff JW, Angeli-Greaves M, Seidel T, Roth G, Volkert F, Ebbinghaus R, Prager HM, Bolt HM, Falkenstein M, Zimmermann A, Klein T, Reckwitz T, Roemer HC, Hartel M, Weistenhöfer W, Schöps W, Rizvi SA, Aslam M, Bánfi G, Romics I, Ickstadt K, Hengstler JG, Golka K.Rs11892031[A] on chromosome 2q37 in an intronic region of the UGT1A locus is associated with urinary bladder cancer risk.ArchToxicol. 2012 Sep;86(9):1369-78. doi: 10.1007/s00204-012-0854-y. Epub 2012 Apr 25.

29. Ovsiannikov $\mathrm{D}^{1}$, Selinski S, Lehmann ML, Blaszkewicz M, Moormann O, Haenel MW, Hengstler JG, GolkaK.Polymorphic enzymes, urinary bladder cancer risk, and structural change in the local industry. JToxicolEnvironHealth A. 2012;75(8-10):557-65. doi: 10.1080/15287394.2012.675308.

30. Occupation and bladder cancer in a population-based case-control study in Northern New England. Colt JS, Karagas MR, Schwenn M, Baris D, Johnson A, Stewart P, Verrill C, Moore LE, Lubin J, Ward MH, Samanic C, Rothman N, Cantor KP, Beane Freeman LE, Schned A, Cherala S, Silverman DT. Occup Environ Med. 2011 Apr;68(4):239-49. doi: 10.1136/oem.2009.052571. Epub 2010 Sep 23.

31. CYP1A2 polymorphisms, occupational and environmental exposures and risk of bladder cancer.Pavanello S, Mastrangelo G, Placidi D, Campagna M, Pulliero A, Carta A, Arici C, PorruS.Eur J Epidemiol. 2010 Jul;25(7):491-500. doi: 10.1007/s10654-010-9479-8. Epub 2010 Jun 18. 
32. Prominent bladder cancer risk factors in Iran.Shakhssalim N, Hosseini SY, Basiri A, Eshrati B, Mazaheri M, SoleimanirahbarA.Asian Pac J Cancer Prev. 2010;11(3):601-6.

33. Bladder cancer risk in painters: a meta-analysis.Guha N, Steenland NK, Merletti F, Altieri A, Cogliano V, StraifK.Occup Environ Med. 2010 Aug;67(8):568-73. doi: 10.1136/oem.2009.051565. Review.

34. Bladder cancer among hairdressers: a meta-analysis.Harling M, Schablon A, Schedlbauer G, Dulon M, NienhausA.Occup Environ Med. 2010 May;67(5):351-8. doi: 10.1136/oem.2009.050195. Review.

35. Occupational exposures to polycyclic aromatic hydrocarbons and resiratory and urinary tract cancers: an updated systematic review and a meta-analysis to 2014.Rotta M, Bosetti C, BocciaS,Boffetta P, La VecchiaC. Arch. Toxicol (2014) 88; 1479-1490. DOI 10.1007/s00204-014-1296-5

36. http://www.ifado.de/en/research_applications/central_units/clinical_occupational_medicine/ bladdercancerquestionnaire/Golka K ${ }^{1}$, Abreu-Villaca Y, AnbariAttar R, Angeli-Greaves M, Aslam M, Basaran N, Belik R, Butryee C, Dalpiaz O, Dzhusupov K, Ecke TH, GalambosH,Galambos H, Gerilovica H, Gerullis H, Gonzalez PC, Goossens ME, Gorgishvili-Hermes L, Heyns CF, Hodzic J, Ikoma F, Jichlinski P, Kang BH, KiesswetterE,Krishnamurthi K, Lehmann ML, Martinova I, Mittal RD, Ravichandran B, Romics I, Roy B, Rungkat-Zakaria F, Rydzynski K, Scutaru C, Shen J, SoufiM,Toguzbaeva K, VuDuc T, Widera A, Wishahi M, Hengstler JG.Bladder cancer documentation of causes: multilingual questionnaire, 'bladder cancer doc'.Front Biosci (Elite Ed). 2012 Jun 1; 4:2809-22. 